\title{
Zivilgesellschaft als Arbeitsmarkt
}

\author{
Christian Hohendanner IAB | Jana Priemer Ziviz | \\ Boris Rump DOSB | Wolfgang Schmitt BAGFW
}

Im folgenden Kapitel werden zivilgesellschaftliche Organisationen und ihr Beitrag für den Arbeitsmarkt behandelt. Neben allgemeinen Strukturen werden Entwicklungstendenzen vorgestellt. Die Ergebnisse beruhen auf den Daten des statistischen Unternehmensregisters des Statistischen Bundesamtes, dem IAB-Betriebspanel, den Erhebungen der Bundesarbeitsgemeinschaft der Freien Wohlfahrtspflege, dem ZiviZ-Survey sowie auf Daten des Sportentwicklungsberichts (vgl. Kapitel 8.1).

\subsection{Beschäftigungsvolumen und -entwicklung ${ }^{1}$}

Der Dritte Sektor ${ }^{2}$, beziehungsweise die organisierte Zivilgesellschaft, spielt eine zentrale Rolle für den deutschen Arbeitsmarkt. In den vergangenen zehn Jahren hat sich die Zahl der bezahlten MitarbeiterInnen (sozialversicherungspflichtig und geringfügig Beschäftigte) im Dritten Sektor nach Angaben des IAB-Betriebspanels von 2,9 im Jahr 2007 auf 3,7 Millionen im Jahr 2016 merklich erhöht. ${ }^{3}$ Die Beschäftigungsentwicklung übertraf damit das allgemeine Beschäftigungswachs-

1 Dieses Unterkapitel wurde verfasst von Christian Hohendanner, IAB, Wolfgang Schmitt, BAGFW und Boris Rump, DOSB..

2 Unter dem Dritten Sektor verstehen wir jenen Bereich, der weder dem Staat noch dem Markt oder der Familie zuzuordnen ist. Typischerweise werden eingetragene Vereine, Stiftungen, gemeinnützige GmbHs und ein Teil der Genossenschaften hinzugezählt (vgl. auch Kapitel 3, Abschnitt 3.1).

3 Bei einer umfassenden Betrachtung der Beschäftigung, bei der zusätzlich PraktikantInnen, LeiharbeiterInnen, freie MitarbeiterInnen und TeilnehmerInnen an „Arbeitsgelegenheiten mit Mehraufwandsentschädigung“ (sogenannte „Ein-Euro-Jobber") mitgezählt werden, waren 2016 knapp 3,9 Millionen Personen im Dritten Sektor tätig (2007: 3,3 Millionen). 
Abbildung 5.1 Anzahl bezahlte Mitarbeitende* im Dritten Sektor (in Millionen)

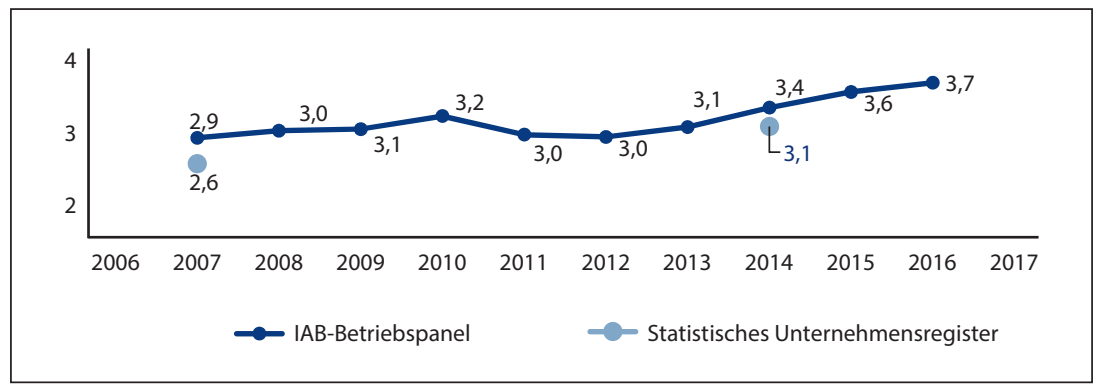

Datengrundlagen: IAB-Betriebspanel 2007-2016 und statistisches Unternehmensregister 2007 und 2014; * IAB-Betriebspanel: sozialversicherungspflichtig und geringfügig Beschäftigte; Unternehmensregister: sozialversicherungspflichtig und geringfügig Beschäftigte ohne Menschen mit Behinderungen in Werkstätten. Im Jahr 2014 waren 347000 Menschen mit Behinderungen in Werkstätten.

tum und der Dritte Sektor konnte seinen Anteil an der Gesamtbeschäftigung leicht ausbauen. Im Jahr 2016 arbeiteten 9,9 Prozent aller sozialversicherungspflichtig und geringfügig Beschäftigten im Dritten Sektor, 2007 waren es noch 9,5 Prozent.

Auch die Daten des statistischen Unternehmensregisters ${ }^{4}$ verdeutlichen, dass zivilgesellschaftliche Organisationen bedeutende Arbeitgeber sind, vor allem als Einrichtungs- und Angebotsträger zentraler Bereiche der täglichen Daseinsvorsorge im Gesundheits- und Sozialwesen. Die Daten belegen zwischen 2007 und 2014 einen deutlichen Anstieg von 2,6 auf fast 3,1 Millionen sozialversicherungspflichtig und geringfügig Beschäftigte (vgl. Abb. 5.1). Anders als bei den Daten des IAB-Betriebspanels sind sozialversicherungspflichtig beschäftigte Menschen mit Behinderungen in Werkstätten in den Zahlen des statistischen Unternehmensregisters nicht enthalten, was die unterschiedlichen Werte der beiden Datenquellen zum Teil erklären dürfte. Im Jahr 2014 waren 347 ooo Menschen mit Behinderungen in Werkstätten beschäftigt, für 2007 gibt es keine Vergleichszahl aus dem Unternehmensregister. Einschließlich der in Werkstätten und der geringfügig beschäftigten Menschen mit Behinderungen liegt die Zahl aller bezahlten Beschäftigten im Dritten Sektor nach Angaben aus dem statistischen Unternehmensregister im Jahr 2014 bei 3,4 Millionen Personen.

4 Den Angaben aus dem statistischen Unternehmensregister liegen alle rechtlichen Einheiten zugrunde, die im Laufe des Berichtsjahres mindestens einen sozialversicherungspflichtig Beschäftigten hatten. Weitere Informationen zum Unternehmensregister siehe Synopse und Steckbrief im Anhang. 
Abbildung 5.2 Entwicklung der Mitarbeitenden* der Einrichtungen und Dienste der Freien Wohlfahrtspflege in den Jahren 2004 bis 2012 (in Tausend)

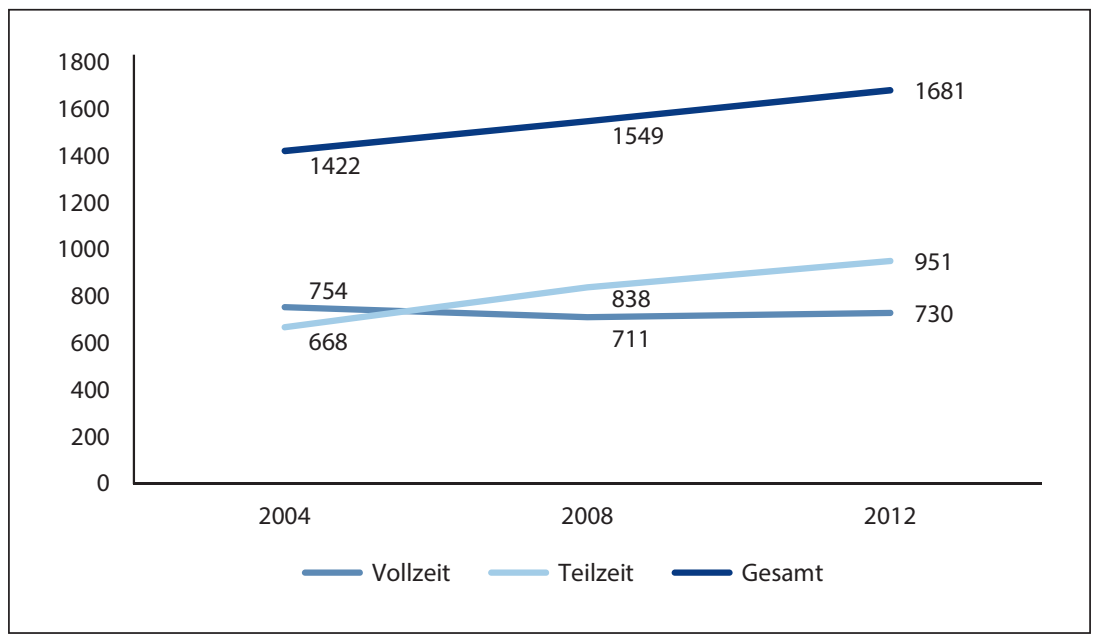

Quelle: Bundesarbeitsgemeinschaft der Freien Wohlfahrtspflege e. V. 2014. *Die Gesamtzahl der Mitarbeitenden schließt die Zahl der hauptamtlich Beschäftigten der Selbsthilfegruppen und der Gruppen des bürgerschaftlichen Engagements mit ein.

Laut statistischem Unternehmensregister arbeiteten im Jahr 2014 gut 2,7 Millionen sozialversicherungspflichtig Beschäftigte (ohne in Werkstätten beschäftigte Menschen mit Behinderungen $)^{5}$ im Dritten Sektor. 2007 waren es noch knapp 2,3 Millionen. Dies entspricht einem Anstieg von 18,4 Prozent. Die Gesamtzahl der sozialversicherungspflichtig Beschäftigten hat sich in diesen sieben Jahren dagegen nur um 11,5 Prozent erhöht. Damit waren 2014 gut 9,7 Prozent aller sozialversicherungspflichtig Beschäftigten im Dritten Sektor tätig (2007: 9,2 Prozent). Hinzu kamen im Jahr 2014 noch die 350 ooo geringfügig Beschäftigten - 2007 waren es rund 300 ooo (vgl. Kapitel 5.4).

Ein Großteil der im Dritten Sektor Beschäftigten ist bei einem Träger der Freien Wohlfahrtspflege (zum Beispiel: Arbeiterwohlfahrt, Deutscher Caritasverband, Der Paritätische Gesamtverband, Deutsches Rotes Kreuz, Diakonie

5 Die 347 ooo Behinderten in Werkstätten im Jahr 2014 sind hier nicht eingerechnet. Einschließlich Behinderten in Werkstätten liegt die Zahl der sozialversicherungspflichtig Beschäftigten in 2014 bei 3050000 . Für 2007 sind aus dem Unternehmensregister keine Angaben zur Zahl der Behinderten in Werkstätten verfügbar. 
Deutschland und Zentralwohlfahrtsstelle der Juden in Deutschland) tätig: In den Einrichtungen und Diensten der Freien Wohlfahrtspflege waren im Jahr 2012 insgesamt knapp 1,7 Millionen Personen hauptamtlich beschäftigt. Auch in der Freien Wohlfahrtspflege zeigt sich im Vergleich zu den früheren Erhebungen ein kontinuierlicher Anstieg der Beschäftigtenzahlen (2004: 1,4 Millionen; 2008: 1,5 Millionen; 2012: 1,7 Millionen). Die Erhebungen des Deutschen Olympischen Sportbundes deuten in eine ähnliche Richtung. So berichten etwa doppelt so viele Sportvereine über einen starken Anstieg bezahlter MitarbeiterInnen (Sportentwicklungsbericht 2015/2016, S. 32-33).

\subsection{Wirtschaftszweige, Aufgabenfelder und Bundesländer ${ }^{6}$}

Aus dem statistischen Unternehmensregister geht hervor, welchen Wirtschaftsbereichen die Unternehmen des Dritten Sektors und deren Beschäftigte zuzuordnen sind. In Abb. 5.3 sind die prozentualen Anteile der im Dritten Sektor sozialversicherungspflichtig beschäftigten Arbeitnehmer nach Wirtschaftszweigen dargestellt.

Im Zeitraum von 2007 bis 2014 gab es keine wesentlichen Verschiebungen bei der Bedeutung des Dritten Sektors als Arbeitgeber innerhalb der verschiedenen Wirtschaftsbereiche. Mit 83,5 Prozent sind die meisten Beschäftigten einer Organisation des Bereichs „Interessenvertretungen, religiöse Vereinigungen“ im Dritten Sektor angestellt. Hierzu gehören beispielsweise Gewerkschaften und andere Interessenverbände sowie Parteien und kirchliche Vereinigungen, die mindestens einen sozialversicherungspflichtig Beschäftigten angestellt haben. Im Gesundheits- und Sozialwesen, dem wichtigsten Beschäftigungsfeld im Dritten Sektor (vgl. Abb. 5.3), sind 42,2 Prozent aller sozialversicherungspflichtig Beschäftigten angestellt. Mit ebenfalls anteiligen 41,7 Prozent an allen sozialversicherungspflichtig Beschäftigten ist der Dritte Sektor auch im Bereich „Erziehung und Unterricht"7 ein bedeutender Arbeitgeber. In diesen Bereich fallen unter anderem die Einrichtungen der Kinder- und Jugendhilfe einschließlich Kindergärten und zunehmend auch Schulen in freier Trägerschaft. In der außerhalb von Unternehmen stattfindenden „Forschung und Entwicklung“ in eigenständigen Instituten stellen zum Dritten Sektor zählende Einrichtungen 23,2 Prozent der ArbeitnehmerInnen. Im

6 Dieses Unterkapitel wurde verfasst von Jana Priemer, ZiviZ, Christian Hohendanner, IAB und Wolfgang Schmitt, BAGFW.

7 Insbesondere bei Erziehung und Unterricht ist zu berücksichtigen, dass die bei öffentlichen Einrichtungen tätigen Beamten keine sozialversicherungspflichtig Beschäftigten sind. Auf sozialversicherungspflichtig Beschäftigte und Beamte bezogen ist der Anteil der im Dritten Sektor Beschäftigten mit rund 23 Prozent deutlich geringer. 
Abbildung 5.3 Anteil der sozialversicherungspflichtig Beschäftigten des Dritten Sektors an der Gesamtbeschäftigung im Wirtschaftsbereich (in Prozent)

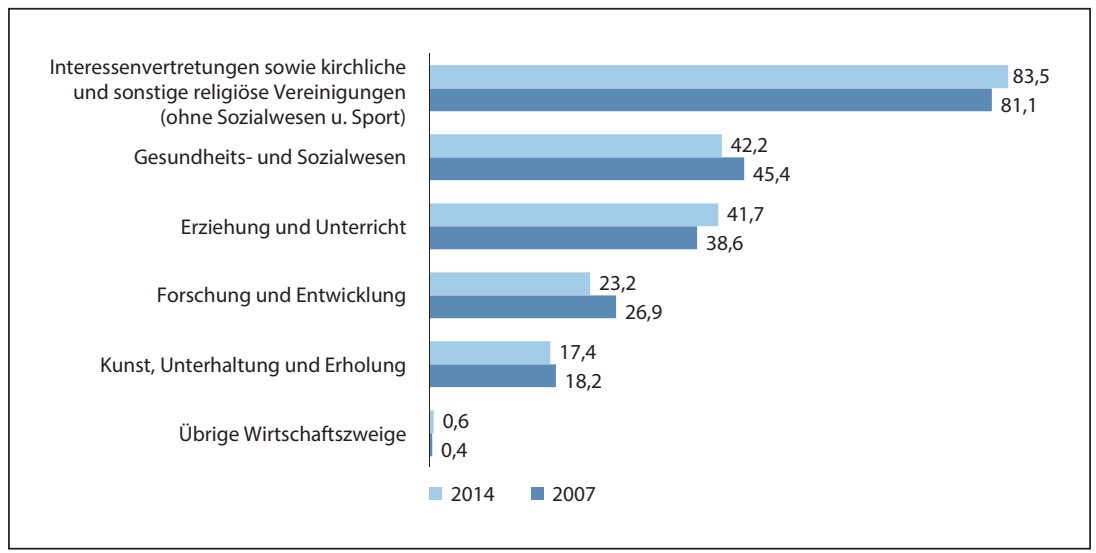

Quelle: Statistisches Unternehmensregister 2007 und 2017. Die Angaben sind ohne sozialversicherungspflichtig beschäftigte Menschen, Menschen mit Behinderungen in Werkstätten und geringfügig Beschäftigte. Da Menschen mit Behinderungen in Werkstätten 2007 nicht zugeordnet werden konnten, wurden sie im Jahr 2014 aus Vergleichsgründen modellhaft herausgerechnet.

Wirtschaftszweig „Kunst, Unterhaltung und Erholung“, zu denen unter anderem Museen sowie andere Kultur- und Freizeiteinrichtungen in gemeinnütziger Trägerschaft gehören, sind rund 17,4 Prozent der Beschäftigten dem Dritten Sektor zuzuordnen.

Die absolute Zahl der sozialversicherungspflichtig Beschäftigten im Dritten Sektor hat sich in allen fünf Wirtschaftsbereichen im Vergleich zu 2007 erhöht. 61,o Prozent aller Beschäftigten waren im Jahr 2014 im „Gesundheits- und Sozialwesen“ tätig (Abb. 5.4). Dies sind 1,65 Millionen Beschäftigte (2007: 1,41 Millionen). An zweiter Stelle folgen „Interessenvertretungen und religiöse Vereinigungen“. Im Jahr 2014 arbeiteten dort 450000 ArbeitnehmerInnen (2007: 410 000). Im Bereich „Erziehung und Unterricht“ waren 2014 rund 390000 Sozialversicherungspflichtige beschäftigt (2007: 310 ooo). Die verbleibenden 8,o Prozent der Beschäftigten des Dritten Sektors verteilen sich über verschiedene Wirtschaftsbereiche, wobei nur in den Bereichen „Kunst, Unterhaltung und Erholung“ sowie im Bereich „Forschung und Entwicklung“ der Dritte Sektor in Relation zu allen dort Beschäftigten ein nennenswertes Gewicht hat.

Die Organisationen des Dritten Sektors nehmen nicht in allen Bundesländern denselben Stellenwert als Arbeitgeber ein (Abb. 5.5). Während im Jahr 2014 
Abbildung 5.4 Verteilung der sozialversicherungspflichtig Beschäftigten des Dritten Sektors nach Wirtschaftszweigen 2014 (in Prozent)

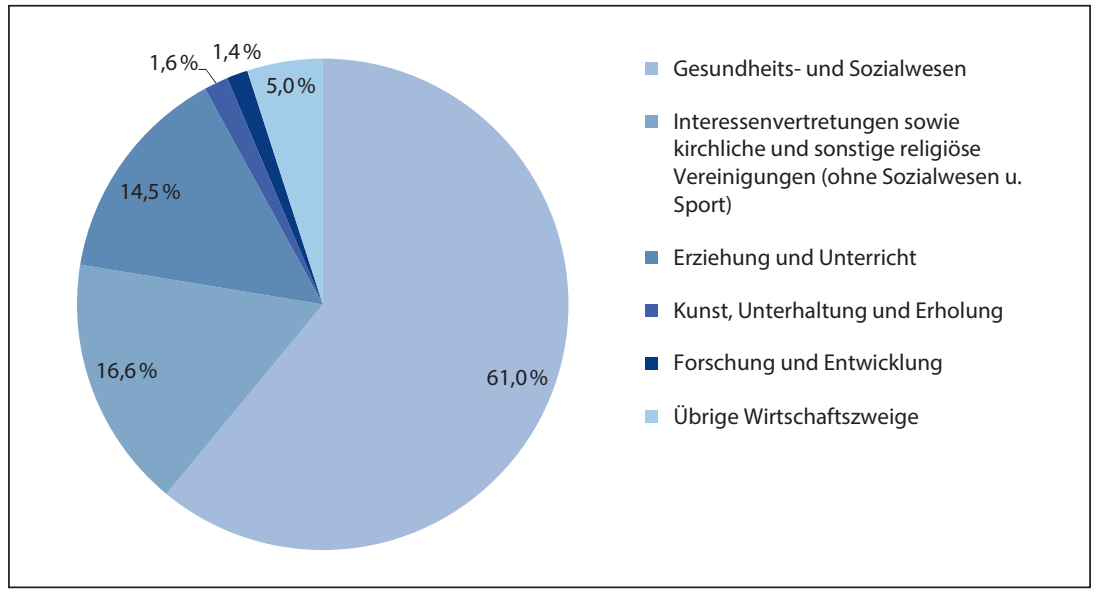

Quelle: Die Berechnungen basieren auf dem statistischen Unternehmensregister des Statistischen Bundesamtes. Die Angaben sind ohne sozialversicherungspflichtig beschäftigte Menschen, Menschen mit Behinderungen in Werkstätten und ohne geringfügig Beschäftigte. Menschen mit Behinderungen in Werkstätten wurde modellhaft herausgerechnet.

Abbildung 5.5 Anteile der sozialversicherungspflichtig Beschäftigten ${ }^{1}$ des Dritten Sektors innerhalb der Bundesländer 2014 (in Prozent)

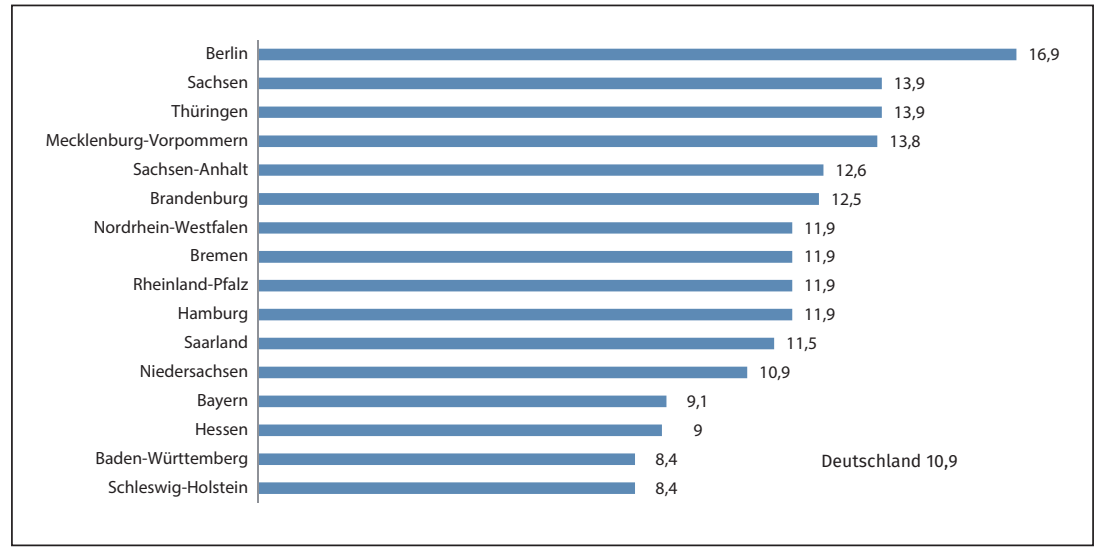

1) Bei den sozialversicherungspflichtig Beschäftigten sind Menschen mit Behinderungen in Werkstätten mit eingeschlossen. Geringfügig Beschäftigte sind nicht enthalten. 
in Berlin 16,9 Prozent aller sozialversicherungspflichtig Beschäftigten bei einer Organisation der Zivilgesellschaft oder bei einer von einem zivilgesellschaftlichen Träger geführten Einrichtung tätig waren, liegt der Vergleichswert in Baden Württemberg und Schleswig Holstein lediglich bei 8,4 Prozent. Auch Bayern und Hessen weisen unterdurchschnittliche Werte auf. Dagegen liegen alle fünf Flächenstaaten aus den neuen Ländern deutlich über dem gesamtdeutschen Durchschnitt.

Die Ursachen für diese Unterschiede dürften vielfältig sein. So scheint die Bedeutung des Dritten Sektors als Arbeitgeber in wirtschaftlich eher schwächeren Bundesländern höher zu sein als in wirtschaftlich besser gestellten Regionen. Der Bedarf an wohlfahrtsstaatlichen Leistungen und damit an Dritt-Sektor-Organisationen dürfte in Regionen mit hoher Arbeitslosigkeit und einer eher alternden Bevölkerung ausgeprägter sein. In städtisch geprägten Bundesländern wie Nordrhein-Westfalen und den Stadtstaaten dürften wiederum strukturelle Aspekte wie die Konzentration von Einrichtungen aus den Bereichen Interessenvertretungen, Gesundheits- und Sozialwesen, Erziehung und Unterricht und den anderen typischen Feldern des Dritten Sektors eine größere Rolle spielen. Berlin kommt zusätzlich durch die Hauptstadtfunktion mit allen um die Politik herum angesiedelten Organisationen und Vereinigungen eine Sonderrolle zu.

Die Einteilung nach Wirtschaftszweigen liefert nur bedingte Einblicke in die konkreten Aufgabenfelder von Organisationen des Dritten Sektors, weshalb am Beispiel der Freien Wohlfahrtspflege ein Bereich differenziert nach Arbeitsfeldern betrachtet wird. Dazu wird die Statistik der Freien Wohlfahrtspflege aus dem Jahr 2012 herangezogen (vgl. Abb. 5.6). Mit 26,5 Prozent aller Beschäftigten stellt der Altenhilfebereich das größte Arbeitsfeld der Wohlfahrtspflege dar: Mehr als ein Viertel der Beschäftigten - oder 445000 Arbeitskräfte - sind in diesem Segment tätig. An zweiter Stelle steht die Gesundheitshilfe mit 392 ooo Beschäftigten (23,3 Prozent). Drittgrößter Bereich ist die Kinder- und Jugendhilfe mit 363000 Mitarbeitenden (21,6 Prozent), gefolgt von der Behindertenhilfe mit 317 ooo Personen (18,9 Prozent) und den weiteren Hilfen mit 61 ooo Beschäftigten (3,6 Prozent). Die Familienhilfe und die Hilfen für Personen in besonderen sozialen Situationen sind mit 1,9 bzw. 2,3 Prozent vertreten.

Die Beschäftigungszahlen haben sich in den einzelnen Arbeitsfeldern der Freien Wohlfahrtspflege unterschiedlich entwickelt (vgl. Abb. 5.7). Während bei den Hilfen für Personen in besonderen sozialen Situationen die Zahl der Mitarbeitenden zwischen 2004 und 2012 um 49,8 Prozentpunkte zugenommen hat, lagen die Wachstumsraten bei der Jugendhilfe bei 32,o Prozent, der Behindertenhilfe bei 30,5 Prozent, der Altenhilfe bei 21,1 Prozent und der Gesundheitshilfe bei 6,5 Prozent. Lediglich in der Familienhilfe ist eine Abnahme der Beschäftigtenzahl von 53,3 Prozent festzustellen. 
Abbildung 5.6 Mitarbeitende der Einrichtungen und Dienste der Freien Wohlfahrtspflege nach Arbeitsfeldern im Jahr 2012 (in Prozent)

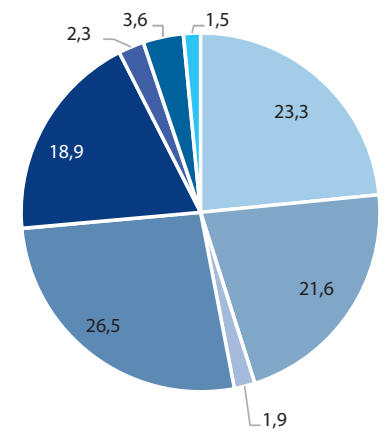

Gesundheitshilfe

Jugendhilfe

Familienhilfe

Altenhilfe

Behindertenhilfe

Hilfe für Personen in besonderen sozialen Situationen

Weitere Hilfen

Aus-, Fort- und Weiterbildungsstätten für soziale und pflegerische Berufe

Quelle: Bundesarbeitsgemeinschaft der Freien Wohlfahrtspflege e.V. 2014.

*Die Gesamtzahl der Mitarbeitenden schließt die Zahl der hauptamtlich Beschäftigten der Selbsthilfegruppen und der Gruppen des bürgerschaftlichen Engagements mit ein.

Abbildung 5.7 Entwicklung der Mitarbeitenden der Einrichtungen und Dienste der Freien Wohlfahrtspflege nach Arbeitsfeldern in den Jahren 2004, 2008 und 2012 (in Tausend)

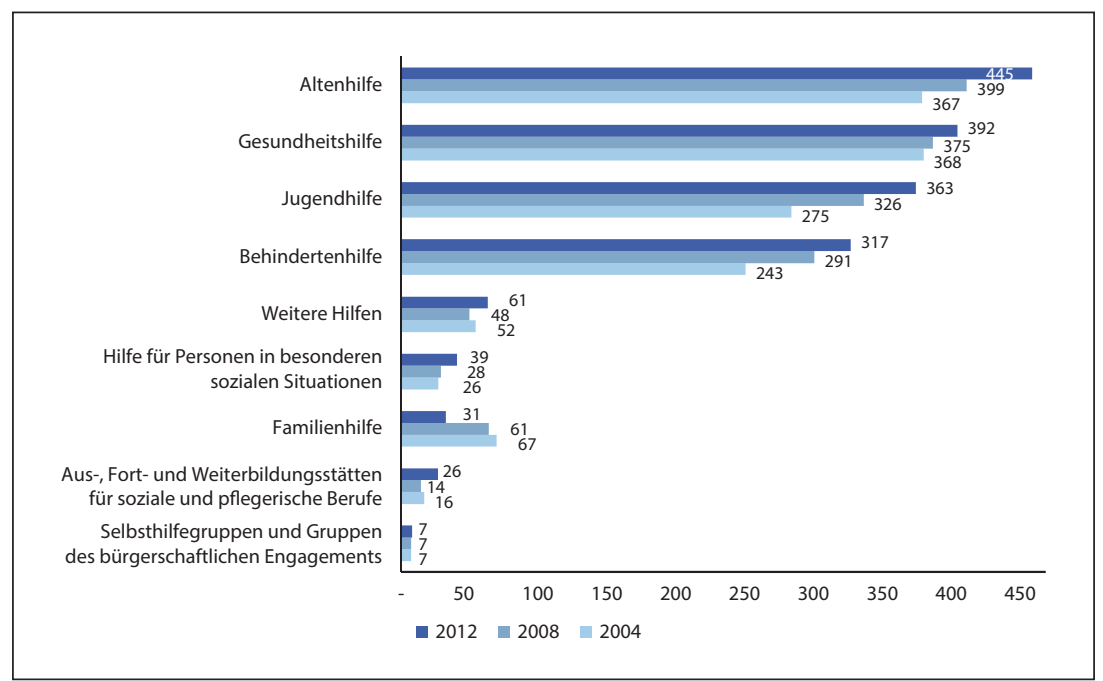

Quelle: Bundesarbeitsgemeinschaft der Freien Wohlfahrtspflege e.V. 2014. *Die Gesamtzahl der Mitarbeitenden schließt die Zahl der hauptamtlich Beschäftigten der Selbsthilfegruppen und der Gruppen des bürgerschaftlichen Engagements mit ein. 
Auch die Daten des ZiviZ-Surveys bestätigen den generellen Trend zu mehr Beschäftigung in zivilgesellschaftlichen Organisationen. Die Zahl der Organisationen ist in den vergangenen Jahren stetig gestiegen (vgl. Kapitel 3). Es stehen also mehr potenzielle Arbeitgeber zur Verfügung. Gleichzeitig ist der Anteil jener Organisationen, in denen es bezahlte Beschäftigung gibt, ebenfalls gestiegen (vgl. Abb. 5.9). Zwischen 2012 und 2017 haben 39,4 Prozent der Organisationen weiteres Personal angestellt. Von rückläufigen Beschäftigtenzahlen berichten nur 6,6 Prozent der Organisationen. Diese Entwicklung lässt sich jedoch nicht in allen Handlungsfeldern gleichermaßen beobachten. In den Organisationen der Sozialen Dienste, im Umwelt- und Naturschutz sowie im Handlungsfeld Bildung und Erziehung wurde bei besonders vielen Organisationen zusätzlich bezahltes Personal eingestellt (vgl. Abb. 5.8).

Abbildung 5.8 Entwicklung der Zahl der bezahlten Beschäftigten seit 2012 nach Handlungsfeld* (in Prozent)

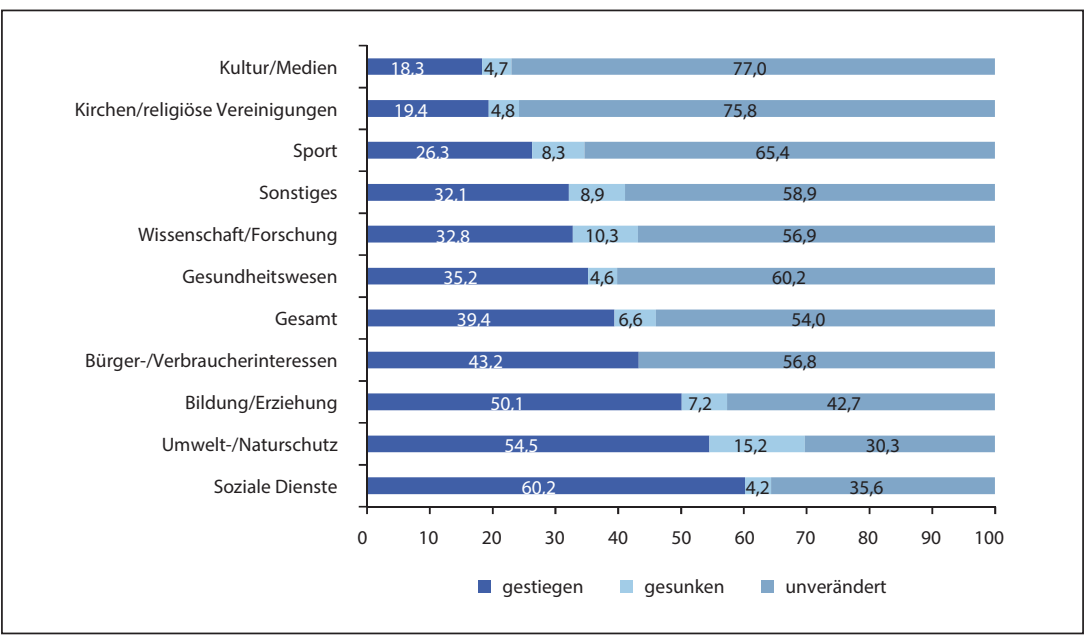

Quelle: ZiviZ-Survey 2017. Nur Organisationen mit bezahlten Beschäftigten, N=1699 (gewichtet), fehlend: 94 (* ohne Bevölkerungs-/Katastrophenschutz, Freizeit/Geselligkeit, Gemeinschaftliche Versorgungsaufgaben, Internationale Solidarität und Wirtschafts-/Berufsverbände wurden ausgeschlossen, da Fallzahlen zu gering). 


\subsection{Professionalisierung im Dritten Sektor ${ }^{8}$}

Der Dritte Sektor ist durch Vielfalt geprägt. Die Organisationen bieten ein breites Spektrum an Angeboten und Dienstleistungen an und weisen zum Teil erhebliche Unterschiede hinsichtlich ihrer Strukturmerkmale auf. Längst nicht alle Segmente des Dritten Sektors haben die finanziellen Ressourcen, um Personal anzustellen, weshalb auch nicht alle Organisationen arbeitsmarktrelevant sind. Daher wirkt die Zunahme der Beschäftigtenzahlen auch nicht in alle Teilbereiche des Dritten Sektors hinein, was die Daten des ZiviZ-Surveys zeigen. Nur einige wenige Handlungsfelder sind hinsichtlich ihres Personals professionell aufgestellt, allen voran die Sozialen Dienste und das Gesundheitswesen - also jene Handlungsfelder, in denen ein Großteil der Aktivitäten der Wohlfahrtsverbände zu verorten ist (vgl. Abb. 5.9).

Der überwiegende Teil der Organisationen arbeitet, mit Ausnahme der meisten gemeinnützigen $\mathrm{GmbHs}$, nach wie vor auf rein ehrenamtlicher Basis. Nicht einmal jede zehnte gemeinnützige $\mathrm{GmbH}$ (7,9 Prozent) kommt ohne bezahltes Personal aus. Diesbezüglich hat sich seit 2012 wenig geändert. Gänzlich anders sieht es bei Vereinen und Stiftungen aus, von denen noch immer die meisten ausschließlich mit Ehrenamtlichen arbeiten, trotz des leichten Trends in Richtung Professionalisierung. 26,9 Prozent der eingetragenen Vereine haben seit 2012 weiteres Personal angestellt (gegenüber 18,7 Prozent im Jahr 2012). Bei den Stiftungen hat sich der Anteil von 29,1 auf 32,2 Prozent erhöht. Doch in der Regel haben sie nur ein oder zwei Beschäftigte, die zudem meist nur in Teilzeit angestellt sind. Etwa jede vierte Stiftung (27,3 Prozent) und jeder vierte Verein (24,3 Prozent) beschäftigt nur jeweils eine bezahlte Mitarbeiterin bzw. einen bezahlten Mitarbeiter. Nur etwa jede zehnte Stiftung (10,5 Prozent) hat 50 oder mehr bezahlte Beschäftigte. Bei den Vereinen sind es sogar nur 7,o Prozent. Insgesamt verzeichnen nur einige wenige Segmente beachtliche Zuwächse.

Erhebliche Unterschiede bestehen auch zwischen den Handlungsfeldern. Vor allem in den Handlungsfeldern, in denen es besonders viele Organisationen gibt, die den Wohlfahrtsverbänden angeschlossen sind und sozialstaatliche Aufgaben übernehmen, arbeitet häufig bezahltes Personal. Dies betrifft jede zweite Organisation der Sozialen Dienste (56,5 Prozent). Auch im Gesundheitswesen (46,5 Prozent) und im Handlungsfeld Bildung und Erziehung (41,1 Prozent) gibt es vergleichsweise viele Organisationen mit bezahltem Personal. In allen drei Handlungsfeldern wurde seit 2012 zusätzliches Personal eingestellt. Doch auch hier gilt, dass noch immer ein großer Teil (jeweils etwa jede zweite Organisation) rein ehrenamtlich arbeitet, denn nicht alle Organisationen dieser Handlungsfelder sind 


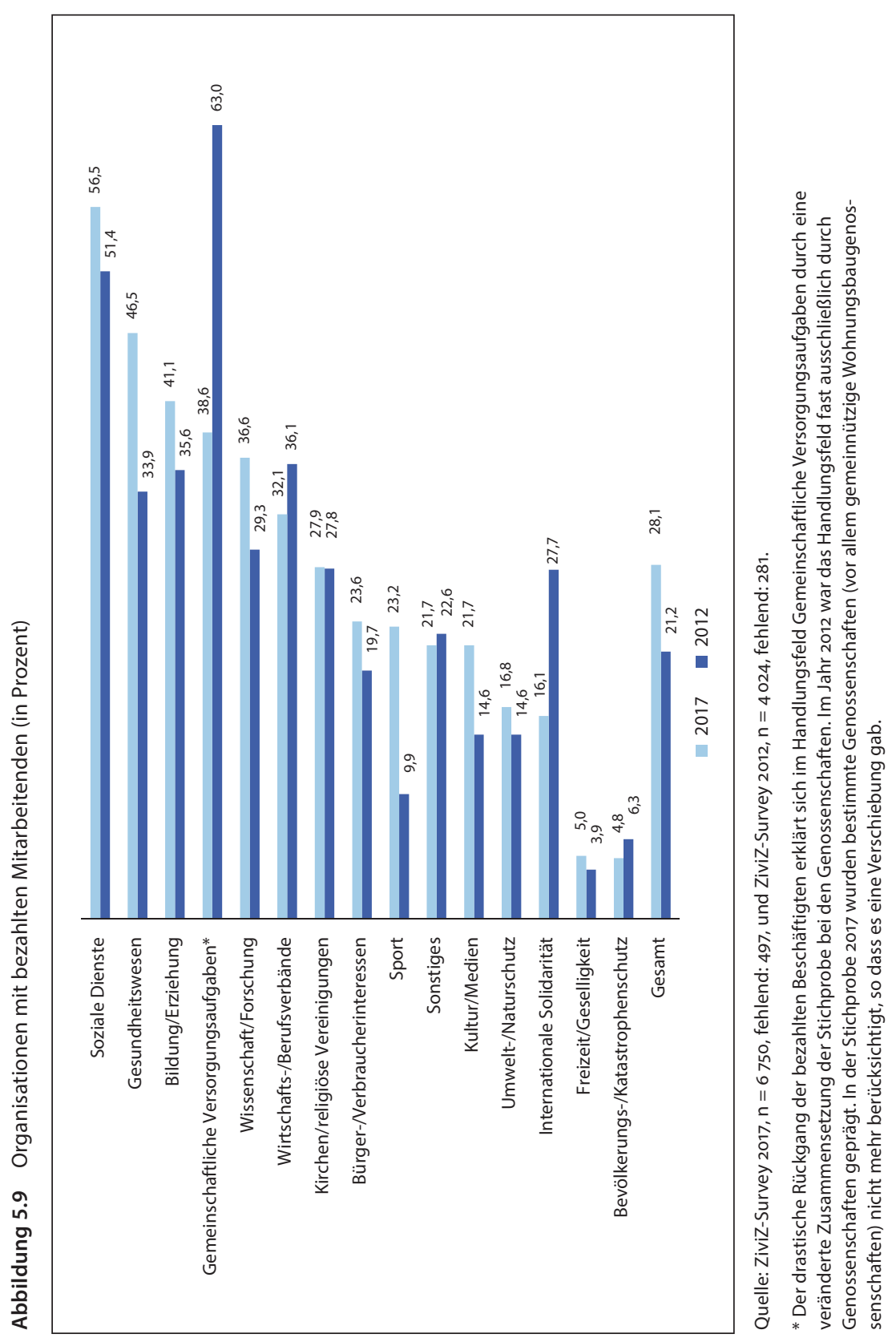


Einrichtungen der Wohlfahrtsverbände. Im Handlungsfeld Bildung gibt es beispielsweise zahlreiche kleine Vereine, die unterschiedlichsten Bildungsaktivitäten nachgehen (zum Beispiel Hausaufgabenbetreuung oder Nachhilfepatenschaften), und vor allem auch viele Fördervereine, die in der Regel ebenfalls ausschließlich von ehrenamtlicher Arbeit getragen werden. Solche kleinen Vereine und Fördervereine gibt es auch in den Sozialen Diensten und im Gesundheitswesen.

Hervorzuheben sind die bemerkenswerten Veränderungen im Sportbereich. Während laut ZiviZ-Survey im Jahr 2012 nur jeder zehnte Sportverein (9,9 Prozent) Personal angestellt hatte, beschäftigt heute fast jeder vierte Sportverein (23,2 Prozent) bezahltes Personal (vgl. Abb. 5.9). Dieser Trend zeichnet sich auch in den jüngsten Daten des Sportentwicklungsberichts ab, die ebenfalls auf einen Anstieg bezahlter Führungspositionen hinweisen. 5,7 Prozent der Sportvereine (5 100 Vereine) haben mindestens eine bezahlte Führungsposition (zum Beispiel bezahlte GeschäftsführerInnen). In 72,6 Prozent dieser Vereine (3 700), werden Führungstätigkeiten in Teilzeit ausgeübt (Breuer und Feiler, 2017, S. 32). In der Privatwirtschaft verhält es sich umgekehrt. Lediglich jede zehnte Führungsposition in privaten Betrieben wird von Teilzeitbeschäftigten besetzt (Kohaut und Möller 2016).

\subsection{Kontinuität und Wandel der Erwerbsformen im Dritten Sektor ${ }^{9}$}

In den letzten Jahren wurde die arbeitsmarktpolitische Bedeutung unterschiedlicher Erwerbsformen zwischen notwendiger Flexibilisierung und problematischer Prekarisierung öffentlich kontrovers diskutiert. Die fortbestehende Relevanz von Teilzeitbeschäftigung, Befristungen, Leiharbeit, geringfügiger Beschäftigung oder freier Mitarbeit auf Basis von Werk- oder Dienstverträgen verdeutlicht zunächst eines: Das deutsche Beschäftigungssystem zeichnet sich durch eine hohe Pluralität der Erwerbsformen aus. Diese hohe Vielfalt der Erwerbsformen zeigt sich auch im Dritten Sektor. In Bezug auf Teilzeit und befristete Arbeitsverträge ist der Dritte Sektor sogar Spitzenreiter.

Teilzeitbeschäftigung: Etwa die Hälfte der im Dritten Sektor Beschäftigten arbeitet in Teilzeit (vgl. Abb. 5.10). Der Teilzeitanteil liegt damit um etwa 20 Prozentpunkte höher als in der Gesamtwirtschaft. Der Teilzeitanteil im Dritten Sektor hat sich parallel zur Entwicklung in der Gesamtwirtschaft in den vergangenen zehn Jahren um 7 Prozentpunkte erhöht. In der Freien Wohlfahrtspflege lag der Teilzeitanteil im Jahr 2012 sogar bei 57 Prozent (vgl. Abb.5.2).

9 Dieses Unterkapitel wurde verfasst von Christian Hohendanner, IAB. 
Abbildung 5.10 Teilzeitbeschäftigung* im Dritten Sektor im Vergleich zur Gesamtwirtschaft (in Prozent)

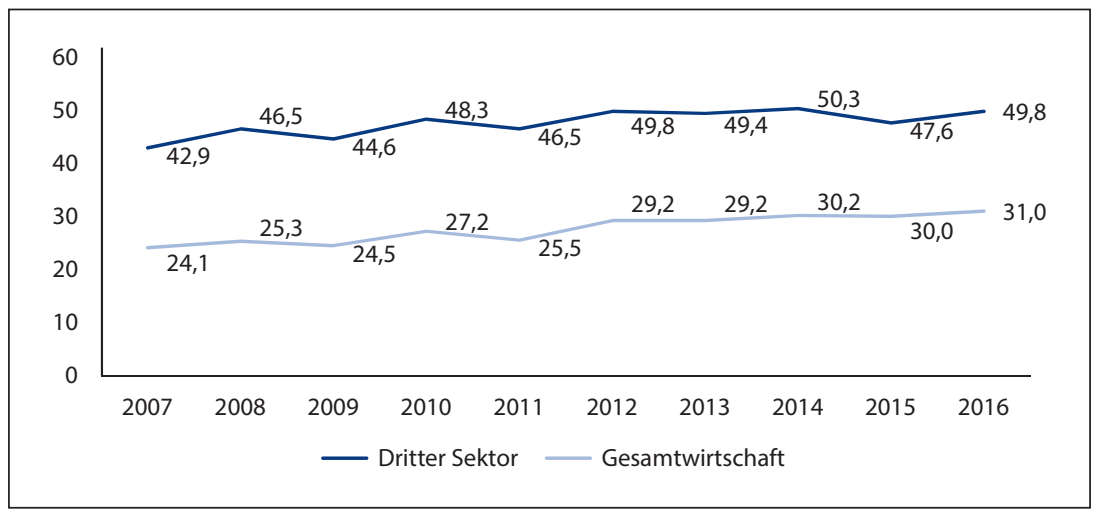

Quelle: IAB-Betriebspanel 2007-2016, hochgerechnete Werte.

* Anteil der Teilzeitbeschäftigten an der betrieblichen Gesamtbeschäftigung.

* Im Gegensatz zu Erhebungen des Statistischen Bundesamtes beruht die Definition der Teilzeitbeschäftigung nicht auf einer bestimmten Stundenzahl (zum Beispiel < 20h), sondern auf der Einteilung der befragten Betriebe in Voll- und Teilzeit.

Teilzeitbeschäftigung ist kein Spezifikum des Dritten Sektors, sondern allgemein von sozialen Dienstleistungen: In den Branchen Erziehung und Unterricht sowie Gesundheit und Soziales lag der Teilzeitanteil nach Angaben des IAB-Betriebspanels in der Gesamtwirtschaft im Jahr 2016 bei 49,o Prozent, in den gemeinnützigen Einrichtungen dieser Branchen bei 52,1 Prozent (nicht abgebildet).

Eine Erklärung für die hohe Teilzeitquote im Dritten Sektor ist der hohe Frauenanteil, der in den letzten zehn Jahren konstant bei etwa 70 Prozent lag. Die hohe Frauenquote im Dritten Sektor lässt sich auch mit einer anderen Statistik verdeutlichen: 14,3 Prozent aller erwerbstätigen Frauen und nur 5,6 Prozent aller erwerbstätigen Männer in Deutschland arbeiteten nach Angaben des IAB-Betriebspanels 2016 im Dritten Sektor.

Geringfügige Beschäftigung: Die geringfügige Beschäftigung ist im Dritten Sektor nicht häufiger verbreitet als in der Gesamtwirtschaft (vgl. Abb. 5.11). Der jüngste Rückgang seit 2014 dürfte auf die Einführung des gesetzlichen Mindestlohns zurückzuführen sein. Durch die Lohnerhöhungen wurde geringfügige Beschäftigung zum Teil in sozialversicherungspflichtige Beschäftigung umgewandelt. 
Abbildung 5.11 Geringfügige Beschäftigung* im Dritten Sektor im Vergleich zur Gesamtwirtschaft (in Prozent)

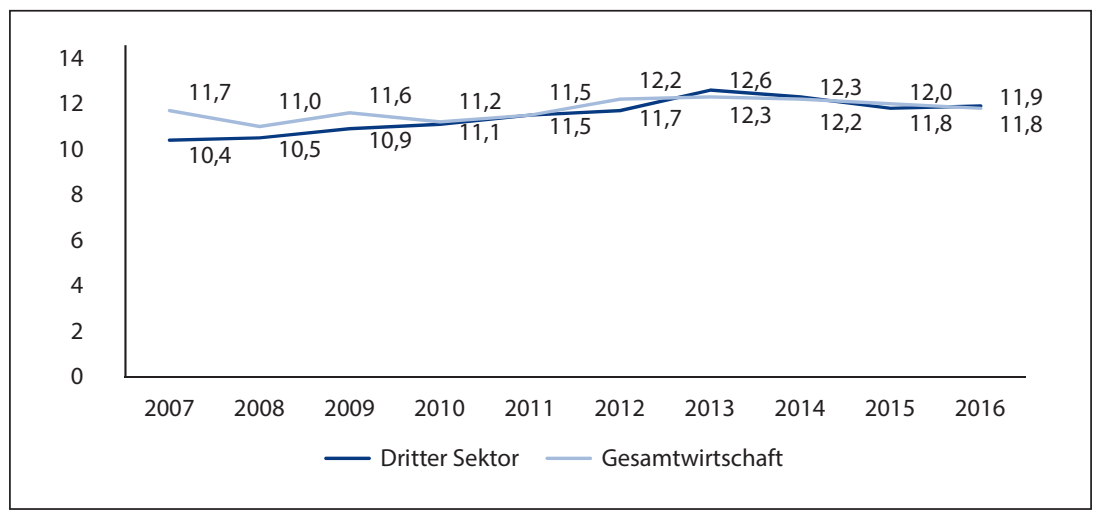

Quelle: IAB-Betriebspanel 2007-2016, hochgerechnete Werte.

* Anteil der geringfügigen Beschäftigung an der betrieblichen Gesamtbeschäftigung.

Befristete Beschäftigung: Die befristete Beschäftigung hingegen ist im Dritten Sektor besonders ausgeprägt. Der Anteil der Befristungen ist, trotz eines leichten Rückgangs seit 2013, mit 15,5 Prozent doppelt so hoch wie in der Gesamtwirtschaft (vgl. Abb. 5.12). Die hohen Anteile befristeter Beschäftigungsverhältnisse im Dritten Sektor lassen sich zum Teil mit der Abhängigkeit von öffentlichen Haushalten und Fördermitteln erklären. Gemeinnützige Projekte werden von der öffentlichen Hand häufig für einen begrenzten Zeitraum von wenigen Jahren finanziert. In einer Befragung von Organisationen des Dritten Sektors aus dem Jahr 2011/12 (Priller et al. 2013) wurden vor allem Mittelkürzungen, finanzielle Planungsunsicherheit sowie strengere Vergabekriterien öffentlicher Mittel als problematische Punkte im Verhältnis zum Staat genannt. Als Gründe für den Einsatz befristeter Arbeitsverträge wurden vor allem befristete Stellenfinanzierungen, der zeitlich begrenzte Personalbedarf sowie die Unsicherheit über die wirtschaftliche Entwicklung der Organisation angegeben (vgl. auch Hohendanner et al. 2015). 
Abbildung 5.12 Befristete Beschäftigung* im Dritten Sektor im Vergleich zur Gesamtwirtschaft (in Prozent)

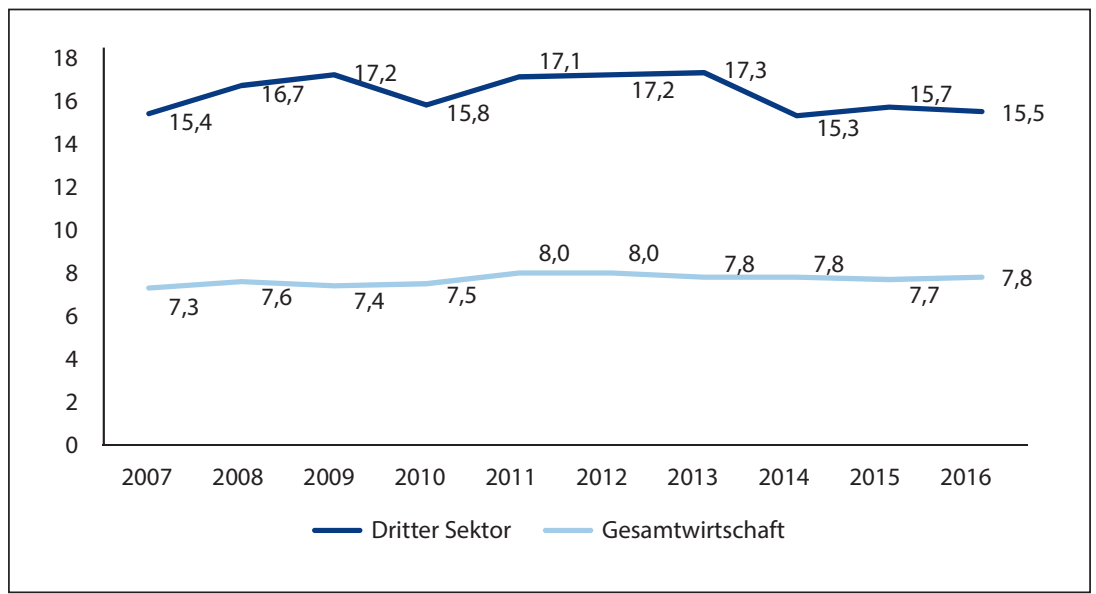

Quelle: IAB-Betriebspanel 2007-2016, hochgerechnete Werte.

* Anteil der befristet Beschäftigten an der betrieblichen Gesamtbeschäftigung (ohne Auszubildende).

Freie Mitarbeit und Leiharbeit: Eine weitere Erwerbsform, die im Dritten Sektor weiter verbreitet ist als in der Gesamtwirtschaft, sind freie MitarbeiterInnen mit Werk- oder Dienstverträgen. 3,3 Prozent aller Beschäftigten im Dritten Sektor sind freie MitarbeiterInnen, in der Gesamtwirtschaft liegt der Anteil lediglich bei 1,9 Prozent (vgl. Abb. 5.13). Vor allem im Bereich Bildung und Erziehung sind vergleichsweise viele Honorarkräfte angestellt (Jambon 2015). Leiharbeit, eine weitere Form der Erwerbsarbeit, spielt hingegen mit 0,3 Prozent im Dritten Sektor nur eine untergeordnete Rolle (vgl. Abb. 5.13). LeiharbeiterInnen werden vor allem im produzierenden Gewerbe eingesetzt, ein Wirtschaftszweig, für den DrittsektorOrganisationen nicht relevant sind. 
Abbildung 5.13 Leiharbeit und freie Mitarbeit im Dritten Sektor im Vergleich zur Gesamtwirtschaft (in Prozent)

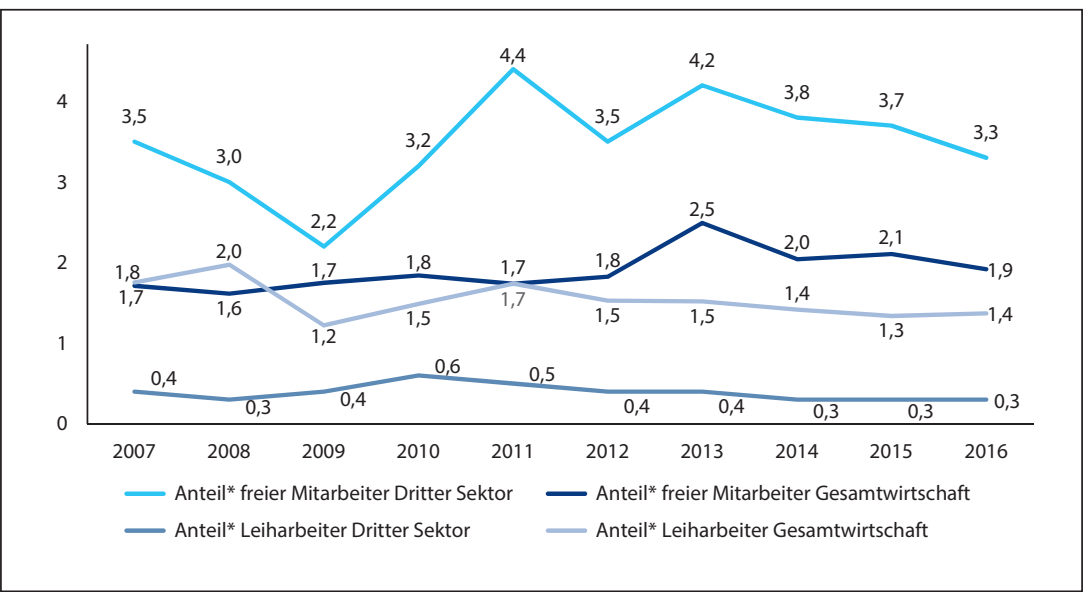

Quelle: IAB-Betriebspanel 2007-2016, hochgerechnete Werte.

*Anteil der freien MitarbeiterInnen/LeiharbeiterInnen an der betrieblichen Gesamtbeschäftigung (jeweils inklusive Leiharbeiterlnnen/freier Mitarbeiterlnnen).

\subsection{Die Rolle des Dritten Sektors für Arbeitsmarktintegration ${ }^{10}$}

Gemeinnützige Organisationen sind nicht nur wichtige Arbeitgeber (jeder zehnte sozialversicherungspflichtig Beschäftigte in Deutschland ist in einer Organisationen des Dritten Sektors beschäftigt), sondern sie übernehmen auch zentrale Funktionen, um Problemen am Arbeitsmarkt entgegenzuwirken. Dadurch tragen die Organisationen dazu bei, die wichtigste Ressource des deutschen Wirtschaftsmodells, nämlich die Beschäftigungsfähigkeit und Qualifikation der Arbeitskräfte, aufrechtzuerhalten und zu verbessern. Das leisten sie vor allem durch unterschiedlichste Weiterbildungs- und Qualifizierungsmaßnahmen, die sich oftmals an besondere Zielgruppen richten, wie zum Beispiel an Menschen mit Migrationshintergrund oder an andere sozial benachteiligte Personen. Mit ihren Angeboten helfen die Akteure des Dritten Sektors diesen Personen bei der Integra-

10 Dieses Unterkapitel wurde verfasst von Jana Priemer, ZiviZ und Christian Hohendanner. 
tion in den ersten oder zweiten Arbeitsmarkt. Neben kommunalen Einrichtungen sind sie heute zentrale „Player“ im Feld der Beschäftigungsförderung für Arbeitslose.

Arbeitsmarktintegration durch Organisationen der Zivilgesellschaft: Von den mehr als 630 ooo Vereinen, Stiftungen, gemeinnützigen GmbHs und Genossenschaften leisten 6,5 Prozent verschiedene Beiträge zur Arbeitsmarktintegration ${ }^{11}$ (Priemer et al. 2017; Labigne et al. 2018). Ihre Angebote sind vielseitig. Sie reichen von spezifischen beruflichen Weiterbildungs- und Qualifizierungsangeboten bis hin zu konkreten Hilfen bei der Integration in den Arbeitsmarkt, zu denen beispielsweise Bewerbertrainings gehören. Werden auch jene Organisationen hinzugezählt, deren Angebote, Initiativen und Dienstleistungen auf Arbeitsmarktintegration im weiteren Sinne abzielen, verdoppelt sich der Anteil sogar auf 11,6 Prozent. Hierbei wurden auch jene Organisationen berücksichtigt, die nicht ausschließlich berufsbezogene, sondern allgemeine Themen betreffende Bildungs- und Qualifizierungsangebote anbieten, wie zum Beispiel Sprachunterricht und Sprachförderung für geflüchtete Menschen.

Tabelle 5.1 Indikatoren zur Arbeitsmarktintegration im ZiviZ-Survey 2017

\begin{tabular}{lll}
\hline Bereich & Teilbereich & Variable* \\
\hline & $\begin{array}{l}\text { Arbeitsmarktintegration } \\
\text { im engeren Sinne }\end{array}$ & "Hilfe bei der Integration in den Arbeitsmarkt" \\
& & $\begin{array}{l}\text { "Berufliche Weiterbildungs- und Qualifizierungs- } \\
\text { angebote“ }\end{array}$ \\
\cline { 2 - 3 } & $\begin{array}{l}\text { Arbeitsmarktintegration } \\
\text { im weiteren Sinne }\end{array}$ & $\begin{array}{l}\text { "Allgemeine Weiterbildungs- und Qualifizierungs- } \\
\text { angebote" }\end{array}$ \\
& & "Sprachunterricht und Sprachförderung" \\
\hline
\end{tabular}

Quelle: Eigene Darstellung.

* Es standen jeweils drei Antwortmöglichkeiten zur Verfügung: oft, manchmal, nie. Es wurden nur jene Organisationen in den Bereich Arbeitsmarktintegration eingeordnet, die "oft" angegeben haben.

11 Die Ergebnisse zur Arbeitsmarktintegration durch zivilgesellschaftliche Organisationen wurde durch das Projekt „Arbeitsmarktintegration - Erfolgsfaktor Zivilgesellschaft“ ermöglicht. Das Projekt fand im Rahmen der Initiative „New Skills at Work“ der JPMorgan Chase Foundation statt. 
Ein-Euro-Jobs: Neben der Bereitstellung von Dienstleistungen ist der Dritte Sektor zudem ein zentrales Einsatzfeld beschäftigungsschaffender Maßnahmen, die überwiegend von der Bundesagentur für Arbeit und von Kommunen implementiert und finanziert werden. Die besondere Leistung des Dritten Sektors zeigt sich beispielsweise in der Beteiligung der Dritter-Sektor-Organisationen am Einsatz von Arbeitsgelegenheiten mit Mehraufwandsentschädigung, besser bekannt als „Ein-Euro-Jobs“. Der Anteil der TeilnehmerInnen im Dritten Sektor hat sich im Zuge des sukzessiven Zurückfahrens der Förderung der Arbeitsgelegenheiten seit dem Jahr 2011 deutlich verringert. Waren im Jahr 2007 5,o Prozent aller Beschäftigten „Ein-Euro-Jobber“, ist dieser Anteil im Jahr 2016 auf 2,2 Prozent zurückgegangen (vgl. Abb. 5.14). Gleichwohl nimmt der Dritte Sektor nach wie vor eine zentrale Rolle beim Einsatz dieser beschäftigungsschaffenden Maßnahmen ein: Knapp die Hälfte aller TeilnehmerInnen an Ein-Euro-Jobs waren über die letzten Jahre in Organisationen des Dritten Sektors tätig.

Abbildung 5.14 Ein-Euro-Jobs* im Dritten Sektor und in der Gesamtwirtschaft (in Prozent)

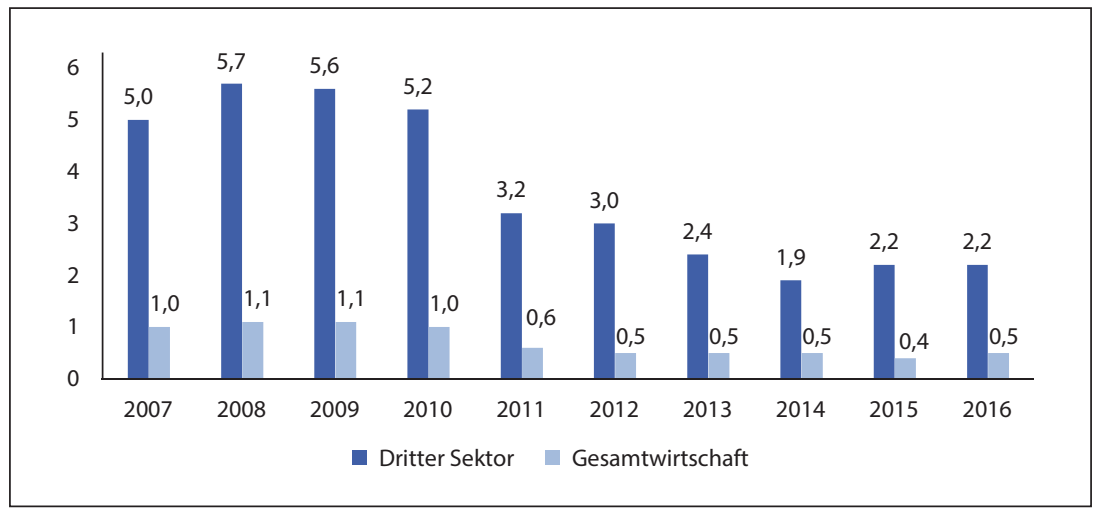

Quelle: IAB-Betriebspanel 2007-2016, hochgerechnete Werte.

*Anteil der Ein-Euro-Jobber an der betrieblichen Gesamtbeschäftigung. 


\subsection{Fazit}

Die organisierte Zivilgesellschaft stellt einen relevanten Faktor im deutschen Arbeitsmarkt dar, der in seiner quantitativen Bedeutung im Vergleich zu anderen Sektoren häufig unterschätzt wird. Mit weit mehr als drei Millionen Beschäftigten arbeiten etwa viermal so viele Personen im Dritten Sektor wie in der Automobilindustrie (im Jahr 2015 laut Volkswirtschaftlicher Gesamtrechnung des Statistischen Bundesamtes rund 870 000). Die Bedeutung zivilgesellschaftlicher Organisationen für den Arbeitsmarkt hat zudem in den letzten Jahren zugenommen. Etwa jede zehnte sozialversicherungspflichtig beschäftigte Person arbeitet in einer Organisation des Dritten Sektors, also nicht in einem gewinnorientierten privatwirtschaftlichen Unternehmen oder in einer staatlichen Einrichtung. Nicht in allen gesellschaftlichen Bereichen hat die Zahl der Beschäftigten in den Organisationen zugenommen. Das Wachstum konzentriert sich vor allem auf jene Bereiche, in denen von den gemeinnützigen Organisationen wohlfahrtsstaatliche Dienstleistungen des Gesundheits- und Sozialwesens erbracht werden.

Zum Beschäftigungszuwachs beigetragen haben dürfte die steigende Zahl an Organisationen des Dritten Sektors sowie die zunehmende Professionalisierung, die mit einer stärkeren Nachfrage nach bezahlten Arbeitskräften in den Organisationen einherging. Die meisten Organisationen - insbesondere Vereine - werden zwar nach wie vor vom ehrenamtlichen Engagement getragen. Doch gerade im Gesundheits- und Sozialwesen, wo bezahlte Arbeitskräfte unerlässlich sind, dürfte der Bedarf an qualifiziertem Fachpersonal demographiebedingt in den nächsten Jahren weiter steigen.

In den Organisationen des Dritten Sektors arbeiten besonders viele Frauen. Der Frauenanteil von 70 Prozent an den Beschäftigten dürften zum Teil auf die große Bedeutung des Gesundheits- und Sozialwesens im Dritten Sektor zurückzuführen sein. Der hohe Frauenanteil erklärt wiederum zum Teil den hohen Anteil an Teilzeitbeschäftigten. Jede zweite im Dritten Sektor beschäftigte Person arbeitet in Teilzeit. Auch befristete Arbeitsverhältnisse und freie MitarbeiterInnen mit Werk- oder Dienstverträgen spielen in einigen Bereichen eine große Rolle. Eine Erklärung für die im Vergleich zur Gesamtwirtschaft überproportionale Bedeutung von Teilzeit, Befristung und freier Mitarbeiter dürften begrenzte Ressourcen, der hohe Kostendruck sowie die Abhängigkeit von staatlichen und privaten Zuwendungen sein, die eine langfristige Personalplanung erschweren.

Die dargestellten Entwicklungen werfen eine Reihe von Fragen auf, die mit einer Darstellung von deskriptiven Zahlenreihen alleine nicht beantwortet werden können: Was sind - neben der erwähnten demografischen Entwicklung - die Treiber des Beschäftigungszuwachses im Dritten Sektor in den letzten Jahren? Warum spielen bestimmte Erwerbsformen eine größere, andere eine geringere 
Rolle? Aus welchen Gründen unterscheidet sich der Anteil bezahlter Mitarbeit zwischen den Bundesländern so deutlich? Um sich der Beantwortung dieser Fragen anzunähern, sind Erhebungen und Analysen hilfreich, die eine Vielzahl potenzieller Einflussfaktoren über einen längeren Zeitraum bei wiederholt befragten Individuen und Organisationen erfassen.

\subsection{Literatur}

Breuer, C., \& Feiler, S. (2017). Sportvereine in Deutschland - Ein Überblick. In C. Breuer (Hrsg.), Sportentwicklungsbericht 2015/2016. Band I. Analyse zur Situation der Sportvereine in Deutschland. (S. 15-46). Hellenthal: Sportverlag Strauß.

Hohendanner, C., Ostmeier, E., \& Ramos Lobato, P. (2015). Befristete Beschäftigung im öffentlichen Dienst. Entwicklung, Motive und rechtliche Umsetzung. IAB-Forschungsbericht 12/2015. Nürnberg.

Jambon, S. (2015). Selbstständige Lehrkräfte im Bildungssektor. In Sozialer Fortschritt, Jg. 64, H. 9/10, S. 247-252.

Kohaut, S., \& Möller, I. (2016). Führungspositionen in der Privatwirtschaft: Im Osten sind Frauen öfter an der Spitze. IAB-Kurzbericht 02/2016. Nürnberg.

Krimmer, H., Priemer, J., \& Labigne, A. (2017): ZiviZ-Survey 2017. Vielfalt verstehen. Zusammenhalt stärken. Berlin.

Labigne, A., Priemer, J., \& Kempf, J. (2018). Erfolgsfaktor organisierte Zivilgesellschaft. Arbeitsmarktintegration in Deutschland. Berlin: Edition Stifterverband.

Priller, E., Alscher, M., Droß, P. J., Paul, F., Poldrack, C. J., Schmeißer, C., \& Waitkus, N. (2013). Dritte-Sektor-Organisationen heute: Eigene Ansprüche und ökonomische Herausforderungen. Ergebnisse einer Organisationsbefragung. WZB Discussion Paper SP IV 2012 - 402. Berlin.

Open Access Dieses Kapitel wird unter der Creative Commons Namensnennung 4.0 International Lizenz (http://creativecommons.org/licenses/by/4.0/deed.de) veröffentlicht, welche die Nutzung, Vervielfältigung, Bearbeitung, Verbreitung und Wiedergabe in jeglichem Medium und Format erlaubt, sofern Sie den/die ursprünglichen Autor(en) und die Quelle ordnungsgemäß nennen, einen Link zur Creative Commons Lizenz beifügen und angeben, ob Änderungen vorgenommen wurden.

Die in diesem Kapitel enthaltenen Bilder und sonstiges Drittmaterial unterliegen ebenfalls der genannten Creative Commons Lizenz, sofern sich aus der Abbildungslegende nichts anderes ergibt. Sofern das betreffende Material nicht unter der genannten Creative Commons Lizenz steht und die betreffende Handlung nicht nach gesetzlichen Vorschriften erlaubt ist, ist für die oben aufgeführten Weiterverwendungen des Materials die Einwilligung des jeweiligen Rechteinhabers einzuholen.

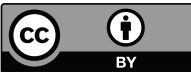

